DOI:

10.1038/nri2124

\title{
Turning on TLR9
}

A new study published in Blood identifies the nuclear protein high-mobility group box 1 protein (HMGB1) as an important modulator of Toll-like receptor 9 (TLR9) activation by CpG-containing DNA (CpG DNA).

CpG DNA motifs in microbial DNA or synthetic oligodeoxynucleotides are known to activate TLR9 in innate immune cells and induce the secretion of pro-inflammatory cytokines. But what is the actual mechanism of TLR9 activation? Ivanov et al. examined the role of HMGB1, an abundant, highly conserved protein that was originally defined as a nuclear transcription factor, but that is now known to be released from cells and act as a chemoattractant for inflammatory cells.

After positively identifying HMGB1 as a CpG-DNA-binding protein, the authors found that HMGB1 enhances TLR9-dependent cytokine responses (specifically interleukin-6 (IL-6), IL-12 and tumour-necrosis factor (TNF) responses) triggered by $\mathrm{CpG}$ DNA. In addition, extracellular HMGB1 acelerates the formation of the CpG-DNA-TLR9 complex.

Having demonstrated interactions between HMGB1 and CpG DNA, and CPG DNA and TLR9, the authors next asked if there could be a direct interaction between HMGB1 and TLR9. Using immunoprecipita-

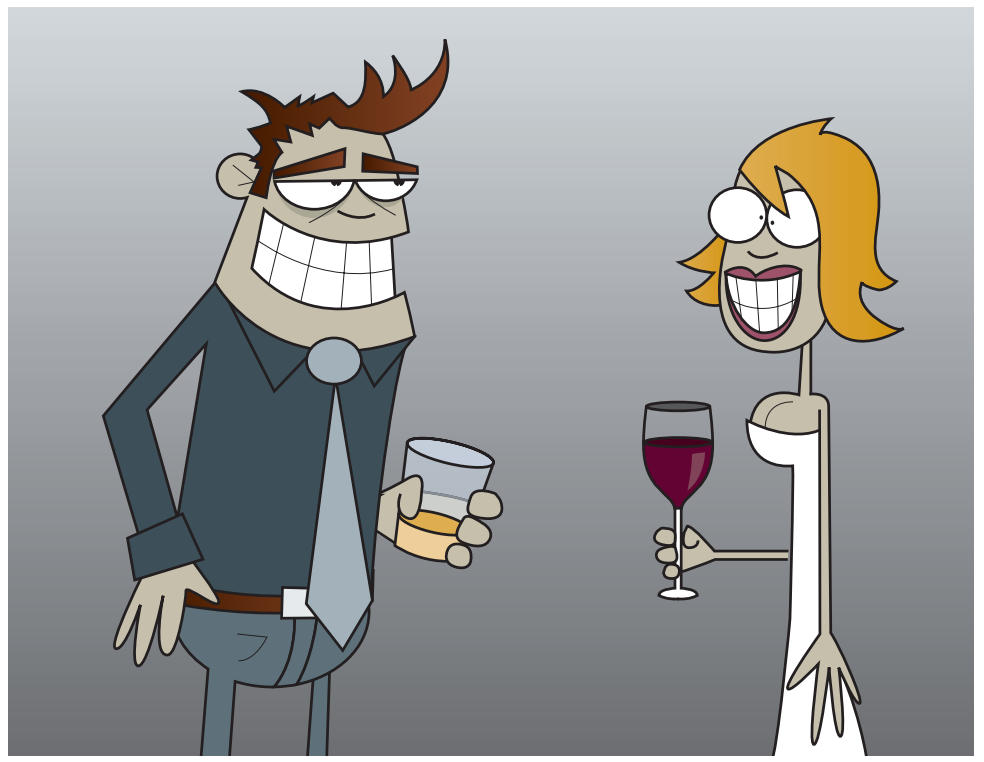

tion and immunofluorescence, they showed that HMGB1 interacts and associates with TLR9, before and during the association of CpG DNA with TLR9, in vesicles that probably belong to the endoplasmic-reticulumGolgi intermediate compartment. HMGB1 does not affect the uptake of CpG DNA or its entry into early endosomes, but rather accelerates the redistribution of TLR9 from the endoplasmic reticulum to the early endosomes in response to CpG DNA.

What happens when HMGB1 is absent from cells? Loss of HMGB1 led to a defect in the response to $\mathrm{CpG}$ DNA in terms of IL-6, IL-12, TNF and inducible nitric-oxide synthase expression. Interestingly, the lack of intracellular TLR9-associated HMGB1 can be compensated by extracellular HMGB1, indicating that HMGB1 can shuttle in and out of immune cells to regulate the inflammatory responses to CpG DNA.

So, HMGB1 has an important role in regulating TLR9 activation by CpG DNA, thereby linking endogenous nuclear proteins and the main family of innate immune sensors in mammals.

Sharon Ahmad

ORIGINAL RESEARCH PAPER Ivanov, S. et al. A novel role for HMGB1 in TLR9-mediated inflammatory responses to $\mathrm{CpG}-\mathrm{DNA}$. Blood 4 June 2007 (doi:10.1182/blood-2006-09-044776) 